\title{
A High Concentration of Polycyclic Aromatic Hydrocarbons in Umbilical Cord Tissue is Associated with An Increased Risk For Fetal Neural Tube Defects
}

\author{
Ying Sun \\ Peking University \\ Shengju Yin ( $\nabla$ sjyin@bjmu.edu.cn ) \\ Peking University \\ Jinhui Yu \\ Peking University \\ Zaiming Su \\ Peking University \\ Mingkun Tong \\ Peking University \\ Yali Zhang \\ Peking University \\ Jufen Liu \\ Peking University \\ Linlin Wang \\ Peking University \\ Zhiwen Li \\ Peking University \\ Aiguo Ren \\ Peking University \\ Lei Jin \\ Peking University
}

\section{Research Article}

Keywords: Polycyclic aromatic hydrocarbons, Umbilical cord, Neural tube defect, Bayesian kernel machine regression, Multiple environmental pollutants

Posted Date: August 13th, 2021 
DOI: https://doi.org/10.21203/rs.3.rs-804705/v1

License: (c) (1) This work is licensed under a Creative Commons Attribution 4.0 International License. Read Full License 


\section{Abstract}

Polycyclic aromatic hydrocarbons (PAHs) are ubiquitous in the environment and have detrimental effects on human health. Embryos are particularly susceptible to environmental insults such as PAHs. We examined the association between prenatal exposure to PAHs and the risk for fetal neural tube defects (NTDs). A case-control study was conducted with 119 NTD cases and 119 controls. A total of 16 PAHs in umbilical cord tissue, determined with gas chromatography-mass spectrometry, were used as in utero exposure markers. Logistic regression and Bayesian kernel machine regression (BKMR) were performed to evaluate the individual and overall effects of PAH exposure on the risk for NTDs, respectively. Median concentrations of 10 PAHs were significantly higher in cases than in controls. In logistic regression, concentrations of four PAHs above the median of all participants were significantly associated with an increased NTD risk, even when potential covariates were adjusted for: phenanthrene, 2.35 -fold (95\% confidence interval [Cl] 1.04-5.34); fluoranthene, 2.37-fold (95\% Cl 1.02-5.48); pyrene, 2.41 -fold (95\% Cl 1.04-5.62); and benzo(b)fluoranthene, 2.95-fold (95\% Cl 1.27-6.86). In BKMR, the risk for NTDs increased when PAH concentrations were all above their 65th percentile compared to their median, while no statistical association between a single compound and NTD risk was observed when the remaining nine PAHs were taken into consideration simultaneously. Together these results show that prenatal PAH exposure is a risk factor for NTDs in offspring.

\section{Introduction}

Polycyclic aromatic hydrocarbons (PAHs) are a class of organic compounds with two or more fused benzene rings (Kim et al., 2013). They are released into the environment by both natural and anthropogenic sources, including the production of organic materials, incomplete burning of fuels, garbage, or other organic substances, leading to the widespread of the PAHs in air, soil, and water (Kim et al., 2013; Stogiannidis and Laane, 2015). Given the ubiquitous of PAHs in the environment, human exposure to PAHs is common. Inhalation, ingestion, and dermal contact are the main exposure route to PAHs (Oliveira et al., 2019).

Studies have suggested that PAHs are far more harmful to human health and the environment than heavy metals and other pollutants (Kamelia et al., 2019; Kim et al., 2013; Stogiannidis and Laane, 2015). Environmental pollutants containing PAHs can cause a variety of damages to human health, such as embryotoxicity, mutagenicity, carcinogenicity, and/or teratogenicity (Drwal et al., 2019; Wang et al., 2015). Moreover, PAHs can interfere with the endocrine system, which can adversely affect the survival and reproduction of animals and humans and the health of their offspring (Bókony et al., 2018; Mark-Kappeler et al., 2011; Waring et al., 2016). Embryos are especially susceptible to environmental pollutants, as their organs are not fully developed, which puts them at a higher risk of developing health problems induced by PAH exposure. Benzo(a)pyrene and benzo(a)pyrene-7,8-dihydrodiol-9,10-epoxide can cause a variety of cardiac and craniofacial malformations in mice, such as gastroschisis, limb deformities, and neural tube defects (NTDs) (Barbieri et al., 1986; Huang et al., 2019a; Huang et al., 2019b; Lin et al., 2018). 
NTDs are a group of common and devastating birth defects that arise from a failure of the neural tube to fuse 3 to 4 weeks after conception (Greene and Copp, 2014). Failed closure in the cranial region (anencephaly) leads to fetal death during pregnancy or at birth (Wilde et al., 2014). Newborns with myelomeningocele or spina bifida have a higher risk of mortality, and those who survive usually face lifelong disability and neurological, cognitive, urological, and digestive complications (Blencowe et al., 2018; Wilde et al., 2014). It has been estimated that 260,100 birth outcomes worldwide were affected by NTDs in 2015 and that $75 \%$ of NTD-affected live births died before age 5 (Blencowe et al., 2018). Therefore, understanding the etiology of NTDs is among the most important public health challenges today. Several risk factors for NTDs, including environmental, nutritional, and genetic factors, have been identified (Copp et al., 2013; Kondo et al., 2017). Recognized environmental risk factors include maternal use of anticonvulsants in early pregnancy, poor nutritional status (e.g., folate deficiency), alcohol consumption, smoking, and exposure to environmental pollutants, among others. (Nikolopoulou et al., 2017). In particular, among environmental pollutants, PAHs are considered to be involved in the development of NTDs, although limited corroborating evidence has been reported to date (Ren et al., 2011).

Epidemiological studies based on small samples have shown that maternal PAH exposure is associated with increased risk for NTDs when PAHs in placental tissue and maternal serum are used as exposure markers (Ren et al., 2011; Wang et al., 2015). However, few studies have examined the association between PAH exposure and risk for NTDs using exposure markers originated in the fetus. The umbilical cord is a structure that connects the mother and the fetus and is responsible for the exchange of materials between them. Therefore, fetal umbilical cord tissue could be a better biospecimen for the assessment of fetal exposure than maternal serum or placental tissue.

In addition, traditional epidemiological studies have reported the association between exposure to individual PAHs and risk for NTDs via multivariable parametric regression approaches and estimated the effects of each compound separately. Recently, experimental and theoretical efforts have been directed at studying the joint effect of multiple environmental pollutants and investigating interactions that may characterize the joint effect of persistent organic pollutants mixtures (Ni et al., 2019; Yin et al., 2021). To the best of our knowledge, no study has investigated the association between PAH mixtures exposure and risk for NTDs. Models that explore the combined effect of PAH mixture on the risk for NTD are necessary. Bayesian kernel machine regression (BKMR) is a new statistical approach, which estimates the potential interactions and nonlinear effects of the mixture components by flexibly modeling the exposure-outcome function (Bobb et al., 2018; Bobb et al., 2015). Given the ubiquitous presence of PAHs in the environment and the health risks associated with their exposure, this study aimed was to examine the association between levels of PAHs in umbilical cord tissue and the risk for NTDs using traditional logistic regression and BKMR.

\section{Materials And Methods}

\subsection{Study design and population}


Detailed information on the study design and population was reported in a previous study (Ren et al., 2011; Yin et al., 2021). In brief, all participants were recruited from Pingding, Zezhou, Taigu, Xiyang, Shouyang counties, and Changzhi city in Shanxi province, China. A woman whose pregnancy, whether live birth, stillbirth, or elective termination, was affected by an NTD was defined as a case; a woman who had a healthy pregnancy and delivered a healthy newborn was defined as a control. NTDs and subtypes were diagnosed by obstetricians based on physical examination after delivery or elective pregnancy termination supplemented by fetal ultrasound. Controls were selected from among healthy deliveries at the same birthing hospital as cases and had the first dates of the last menstrual period close to those of cases ( \pm 4 weeks). All cases and controls lived in the province during the current pregnancy. Ultimately, 119 NTD cases and 119 controls were included in this study. Umbilical cord tissue was collected at delivery or termination of pregnancy and stored at $-20^{\circ} \mathrm{C}$, and then transferred on dry ice to our laboratory and kept at $-20^{\circ} \mathrm{C}$ until analyses. The study was approved by the institutional review board of Peking University, and all participants provided signed consent to participate.

\subsection{Data collection}

Data on maternal characteristics were collected by trained health care workers using a structured questionnaire through face-to-face interviews before the women were discharged from the hospital. The questionnaire included questions about socio-demographic information (i.e., maternal age, occupation, education, parity, reproductive history), lifestyle factors (i.e., alcohol drinking, passive smoking), periconceptional folic acid supplementation, fetal age, and maternal conditions. Gestational age at delivery/termination of pregnancy was determined by the first date of the last menstrual period and the date of delivery or pregnancy termination aided by ultrasound measurement. Fetal gender was recorded at birth or elective termination. Maternal age, gestational age are continuous variables. Alcohol drinking (yes/no), passive smoking (yes/no), periconceptional folic acid supplementation (yes/no), history of birth defects (yes/no), and fever or flu during early pregnancy (yes/no) are dichotomous variables. Alcohol drinking or cigarette smoking at least once a week were classified as having the habit. Women who had ever given birth to a child with malformation were considered to have a history of birth defects. Periconceptional folic acid supplementation was defined as having taken folic acid supplements three months before through three months after conception. Fever or flu during early pregnancy was defined as having a fever or flu during the first trimester of pregnancy.

\subsection{Quantification OF PAHs}

Procedures for preparing umbilical cord tissue and analyzing PAHs were described in detail previously (Ni et al., 2019). Briefly, 16 PAHs were analyzed, naphthalene (NAP), acenaphthylene (ANY), acenaphthene (ACE), fluorene (FLU), phenanthrene (PHE), anthracene (ANT), fluoranthene (FLO), pyrene (PYR), benzo(a)anthracene (BAA), chrysene (CHR), benzo(b)fluoranthene (BBF), benzo(k)fluoranthene (BKF), benzo(a)pyrene (BAP), Indeno(1,2,3-cd)pyrene (I1P), dibenzo(a,h)anthracene (DahA), and benzo(g,h,i)perylene (BghiP). Concentrations of PAHs in umbilical cord tissue were analyzed using gas chromatography (7890, Agilent, Palo Alto, CA, USA) equipped with mass spectrometry (5977A, Agilent). Two method blanks and one quality control sample were analyzed with each batch of samples to 
eliminate any external source of contamination and monitor the condition of gas chromatography-mass spectrometry. The concentration of each PAH was estimated with an individual PAH standard curve. The final PAH concentration of each sample was then calculated by PAH concentration minus the corresponding operation blank. Method recoveries of the $16 \mathrm{PAH}$ standards varied from $36.2 \%$ (ANY) to 103.1\% (PYR), and the detailed data on recovery and detection rates are shown in Table S1. The samples were randomly assigned during the assessment, and the analysts were blind to the status of the samples.

\subsection{Statistical analyses}

Concentrations of PAHs in umbilical cord tissue are presented as $\mathrm{ng} / \mathrm{g}$ dry weight. PAH concentrations below the limit of detection were imputed with half limit of detection. The detection rates for the 16 PAHs ranged from $73.95-100 \%$ in all samples. PAHs with recovery rates less than $60 \%$, that is, NAP, ANY, ACE, FLU, ANT, and DahA were not included in further analyses (Table S1). The 10 PAHs with recovery rates of more than $60 \%$ were included in the final analysis. Non-normal distributions of PAH concentrations in umbilical cord tissue were described with the median and interquartile range.

Significant differences between cases and controls in levels of PAHs were estimated with the Mann Whitney $U$ test. Differences in frequencies of demographic characteristics between cases and controls were evaluated with Pearson's chi-square test or Fisher's exact probability test. Two missing values for gestational weeks in the control group were replaced with the median gestational weeks of the control group. Associations between the concentrations of each PAH and risk for NTDs are presented with odds ratios (ORs) and $95 \%$ confidence intervals (Cls) estimated by unconditional logistic regression. PAH concentrations were defined as "exposed" (above the median) or "referent" (below the median). We adjusted for potential covariates, including maternal age, gestational age at delivery/termination of pregnancy, periconceptional folic acid supplementation, fever or flu during early pregnancy, and passive smoking, related to both NTD risk and concentration of any single PAH in logistic regression analyses.

In addition, we performed BKMR, a novel statistical approach, to evaluate the combined effect of 10 PAHs exposure on the risk for NTDs. BKMR is ideal for estimating the health effects of exposure to multiple pollutants and extracting the posterior inclusion probability of each exposure, which provides an estimate of the importance of the variable in the model and allows the quantification and visualization of both the overall mixture effect and the effects of each mixture component within the context of the overall joint exposure.(Bobb et al., 2018; Bobb et al., 2015). The BKMR model could evaluate the association between exposure to co-pollutants and a binary outcome [(1/0]) variable, which represents whether NTD case or control]. In the circumstances, a probit link function was supposed. The probit BKMR model is given by $\mathrm{Yi}$ $=h\left(P H E, F L O, P Y R, B A A, C H R, B B F, B K F, B A P, I 1 P\right.$, and BghiP) $+\beta^{\top} Z_{i}+e_{i}$. With probit regression in BKMR, where $h($ ) was the exposure-response function and indicated the association among exposures and latent continuous outcomes ( $>0$ is equal to a case, $\leq 0$ is equal to a control), $Z_{i}$ contained a set of covariates, and $\beta$ indicated the effect of the covariates. We modeled the concentrations of PAHs as logtransformed. We adjusted for the same covariates in BKMR as in the adjusted logistic regression model. 
BKMR was conducted in R (version 4.0.2; R Development Core Team) within the RStudio (version 1.2.5042, 2009-2020 RStudio, Inc.) platform. All other statistical analyses were conducted with SPSS 24.0 for windows (IBM, Chicago, IL, USA). Statistical significance was taken as a two-tailed $P<0.05$.

\section{Results}

\subsection{Characteristics of the study participants}

Detailed demographic characteristics of this population are presented in Table 1. Briefly, the frequency of periconceptional folic acid supplementation was lower in NTD cases than in controls, whereas NTD cases had a higher proportion of fever or flu and passive smoking compared to controls. Moreover, NTD cases had a shorter gestational age than controls. Other characteristics such as maternal age, education, fetal gender, parity, history of birth defects, and alcohol consumption showed no significant differences between cases and controls. Characteristics of cases with spina bifida and controls were showed in Table S2; the distribution of all characteristics between the two groups was the same as the total population, except for periconceptional folic acid supplementation, which became statistically non-significant between the two groups. 
Table 1

Characteristics of NTD cases and controls recruited in Shanxi province, China.

\section{Characteristics}

Controls $(n=$

119)

$\mathrm{n}(\%)$
NTDs $(n=$

119)

$\mathrm{n}(\%)$

Age, y

$\begin{array}{llll}<25 & 46(38.7) & 46(38.7) & 0.404 \\ 25-29 & 39(32.8) & 32(26.9) & \\ \geq 30 & 34(28.5) & 41(34.4)\end{array}$

Education

Primary school or less

$7(5.9)$

$12(10.2)$

0.075

Junior high school

$72(60.5)$

80 (68.4)

High school or more

40 (33.6)

25 (21.4)

Fetal gender

Male

Female

Gestational age at delivery/termination of pregnancy,

weeks $(\geq 37)$

Parity $(>1)$

History of birth defects (yes)

Periconceptional folic acid supplementation (yes)

Fever or flu during early pregnancy (yes)

55 (46.6)

$58(52.7)$

0.356

$63(53.4)$

$52(47.3)$

$117(98.3)$

$30(25.2)$

$<$

$54(46.6)$

55 (48.2)

0.797

$1(0.9)$

$5(4.2)$

0.231

59 (52.2)

43 (37.4)

0.024

18 (15.5)

44 (39.3)

$<$

Passive smoking (yes)

89 (75.4)

$94(86.2)$

$10(8.4)$

15 (12.9)

0.260

Abbreviation:

NTD, neural tube defect.

a $P$ value for Pearson's chi-square test or Fisher's exact test.

\subsection{Concentrations of PAHs in controls and cases}

The detection rates of all 16 PAHs were greater than $70 \%$ in all 238 umbilical cord samples, but only 10 PAHs had recovery rates greater than $60 \%$ : one low-molecular-weight PAH (PHE) and nine high-molecular- 
weight PAHs (FLO, PYR, BAA, CHR, BBF, BKF, BAP, I1P, BghiP). Median concentrations of 10 PAHs were all higher in NTD cases than in controls (Table 2). Levels of 10 PAHs were also higher in cases with spina bifida than in controls (Table S3). Given the limited sample sizes for anencephaly and encephalocele subtypes (22 and 10 cases, respectively), we chose not to perform statistical analyses of these two subtypes separately.

Table 2

Concentrations of PAHs in NTD cases and controls enrolled in Shanxi province, China.

\begin{tabular}{|c|c|c|c|}
\hline \multirow[t]{2}{*}{ PAH } & \multicolumn{2}{|c|}{ Median and interquartile range ( $\mathrm{ng} / \mathrm{g}$ dry weight) } & \multirow[t]{2}{*}{$P^{a}$} \\
\hline & Controls $(n=119)$ & NTDs $(n=119)$ & \\
\hline PHE & $12.4(10.4-14.9)$ & $16.8(13.6-22.2)$ & $<0.001$ \\
\hline FLO & $2.74(2.44-3.33)$ & $3.78(3.13-4.85)$ & $<0.001$ \\
\hline PYR & $2.27(1.89-2.80)$ & $3.27(2.47-4.17)$ & $<0.001$ \\
\hline BAA & $0.396(0.314-0.474)$ & $0.546(0.421-0.746)$ & $<0.001$ \\
\hline $\mathrm{CHR}$ & $0.897(0.709-1.370)$ & $1.44(1.10-2.00)$ & $<0.001$ \\
\hline BBF & $0.254(0.155-0.382)$ & $0.387(0.250-0.526)$ & $<0.001$ \\
\hline BKF & $0.127(0.0883-0.189)$ & $0.203(0.112-0.293)$ & $<0.001$ \\
\hline BAP & $0.0856(0.0548-0.133)$ & $0.119(0.0697-0.206)$ & 0.001 \\
\hline I1P & $0.134(0.0864-0.259)$ & $0.184(0.129-0.330)$ & 0.002 \\
\hline BghiP & $0.308(0.262-0.400)$ & $0.443(0.349-0.542)$ & $<0.001$ \\
\hline \multicolumn{4}{|c|}{$\begin{array}{l}\text { Abbreviations: PAH, polycyclic aromatic hydrocarbon; NTD, neural tube defect; PHE, phenanthrene; } \\
\text { FLO, fluoranthene; PYR, pyrene; BAA, benzo(a)anthracene; CHR, chrysene; BBF, benzo(b)fluoranthene } \\
\text { BKF, benzo(k)fluoranthene; BAP, benzo(a)pyrene; I1P, Indeno(1,2,3-cd)pyrene; BghiP, } \\
\text { benzo(g,h,i)perylene. }\end{array}$} \\
\hline
\end{tabular}

\subsection{Individual PAHs and NTD risk: Logistic regression}

We performed unconditional logistic regression analyses to evaluate associations between the concentrations of all individual PAHs and risk for NTDs. As shown in Table 3, increased concentrations of all individual PAHs were associated with elevated NTD risk compared to the reference level (below the median) in the unadjusted model; NTD risk increased 2.35-fold for PHE, 2.37-fold for FLO, 2.41-fold for PYR, and 2.95-fold for BBF after we adjusted for confounders. In the spina bifida subgroup, similar results were obtained with the same adjustment (Table S4, the risk for spina bifida increased 2.51-fold for PHE, 4.23-fold for FLO, 4.02-fold for PYR, and 4.51-fold for BBF. Dose-response analyses suggested that the risk for NTDs increased with an increase in exposure to PAHs. Detailed data on risks for both total NTDs and spina bifida indicated by adjusted ORs of logistic regression can be found in Tables S5 and S6. 
Furthermore, according to the toxic equivalent factors (TEFs) value for PAHs (Nisbet and LaGoy, 1992), we estimated the benzo[a]pyrene equivalent concentration of PAHs and evaluated the comprehensive toxicity of PAHs for the occurrence of NTDs. The risk for NTDs increased when the benzo[a]pyrene equivalent concentration of PAHs above the median compared to the referent (Table S7). Although the results turned to be not significant after adjusting for confounding factors, the directions of the ORs were consistent with the unadjusted ORs. 
Table 3

Concentrations (ng/g dry weight) of PAHs in umbilical cord tissue and risk for NTDs: logistic regression.

\begin{tabular}{|c|c|c|c|c|c|c|c|}
\hline \multirow[t]{2}{*}{ PAHs } & \multirow{2}{*}{$\begin{array}{l}\text { Cutoff } \\
\text { value }^{a}\end{array}$} & \multirow{2}{*}{$\begin{array}{l}\text { Controls } \\
\text { (referent/ } \\
\text { exposure) }\end{array}$} & \multicolumn{5}{|c|}{ Risk for NTDs } \\
\hline & & & $\begin{array}{l}\text { referent/ } \\
\text { exposure }\end{array}$ & $\mathrm{OR}^{\mathrm{b}}(95 \% \mathrm{Cl})$ & $P$ & $\begin{array}{l}\mathrm{aOR}^{\mathrm{c}}(95 \% \\
\mathrm{Cl})\end{array}$ & $P$ \\
\hline PHE & 14.4 & $86 / 33$ & $33 / 86$ & $\begin{array}{l}6.79(3.85- \\
11.98)\end{array}$ & $<.001$ & $\begin{array}{l}2.35(1.04- \\
5.34)\end{array}$ & 0.041 \\
\hline FLO & 3.21 & $86 / 33$ & $33 / 86$ & $\begin{array}{l}6.79(3.85- \\
11.98)\end{array}$ & $<.001$ & $\begin{array}{l}2.37(1.02- \\
5.48)\end{array}$ & 0.044 \\
\hline PYR & 2.71 & $85 / 34$ & $34 / 85$ & $\begin{array}{l}6.25(3.56- \\
11.97)\end{array}$ & $\begin{array}{l}<.001 \\
0.00\end{array}$ & $\begin{array}{l}2.41(1.04- \\
5.62)\end{array}$ & 0.041 \\
\hline BAA & 0.461 & $84 / 35$ & $35 / 84$ & $\begin{array}{l}5.76(3.30- \\
10.06)\end{array}$ & $<.001$ & $\begin{array}{l}2.04(0.89- \\
4.67)\end{array}$ & 0.091 \\
\hline $\mathrm{CHR}$ & 1.17 & $79 / 40$ & $40 / 79$ & $\begin{array}{l}3.90(2.28- \\
6.68)\end{array}$ & $<0.001$ & $\begin{array}{l}1.61(0.72- \\
3.62)\end{array}$ & 0.251 \\
\hline BBF & 0.316 & $77 / 42$ & $42 / 77$ & $\begin{array}{l}3.36(1.98- \\
5.72)\end{array}$ & $<0.001$ & $\begin{array}{l}2.95(1.27- \\
6.86)\end{array}$ & 0.012 \\
\hline BKF & 0.153 & $74 / 45$ & $45 / 74$ & $\begin{array}{l}2.70(1.60- \\
4.57)\end{array}$ & $<.001$ & $\begin{array}{l}1.75(0.78- \\
3.90)\end{array}$ & 0.172 \\
\hline BAP & 0.0971 & $68 / 51$ & $51 / 68$ & $\begin{array}{l}1.78(1.06- \\
2.97)\end{array}$ & 0.028 & $\begin{array}{l}1.09(0.48- \\
2.44)\end{array}$ & 0.842 \\
\hline I1P & 0.172 & $72 / 47$ & $47 / 72$ & $\begin{array}{l}2.35(1.40- \\
3.95)\end{array}$ & 0.001 & $\begin{array}{l}1.94(0.85- \\
4.43)\end{array}$ & 0.116 \\
\hline BghiP & 0.371 & $81 / 38$ & $38 / 81$ & $\begin{array}{l}4.54(2.64- \\
7.84)\end{array}$ & $<$. & $\begin{array}{l}2.19(0.94- \\
5.07)\end{array}$ & 0.069 \\
\hline
\end{tabular}

Abbreviations: PAH, polycyclic aromatic hydrocarbon; NTD, neural tube defect; OR, odds ratio; $\mathrm{Cl}$, confidence interval; PHE, phenanthrene; FLO, fluoranthene ; PYR, pyrene; BAA, benzo(a)anthracene; CHR, chrysene; BBF, benzo(b)fluoranthene; BKF, benzo(k)fluoranthene; BAP, benzo(a)pyrene; I1P, Indeno(1,2,3-cd)pyrene; BghiP, benzo(g,h,i)perylene.

a Median (ng/g dry weight) of all participants.

b Unadjusted OR by binary logistic regression.

${ }^{c}$ Adjusted OR by binary logistic regression, adjusted for maternal age, gestational age at delivery/termination of pregnancy, periconceptional folic acid supplementation, fever or flu during early pregnancy, and maternal passive smoking.

\subsection{Multiple PAHs and NTD risk: BKMR}

Humans are exposed to a wide range of combinations of environmental pollutants that usually comprise multiple highly correlated components. Results of Spearman correlation analyses indicated highly 
significant positive correlations in the concentrations of 10 PAHs (Table S8). We used BKMR, which estimated the joint exposure-response functions for the $10 \mathrm{PAHs}$, to estimate the effects of the PAH mixtures on NTD risk. We obtained the inferences on PAH mixture exposure effects for NTD binary outcome by computing posterior mean estimates (and 95\% posterior credible intervals) using BKMR (Fig. 1). In particular, the model estimated the joint effect of PAH mixture exposure by estimating the expected change (expressed as $\beta_{\text {probit }}$ ) in NTDs associated with concurrent changes in all components of the mixture from their median levels. The risk for NTDs increased when the levels of all 10 PAHs were above their 65th percentile (Fig. 1A). Single-exposure effects were determined to characterize the individual effect of specific PAHs on NTD risk; however, no statistical association between a single compound and NTD risk was observed when the remaining nine PAHs were taken into consideration simultaneously (Fig. 1B). In BKMR, each PAH has a posterior inclusion probability (PIP) ranging from 0 to 1 that can be used to measure the importance of each PAH. All of the 10 PAHs have a PIP over 0.80, while $\mathrm{PHE}, \mathrm{BAA}, \mathrm{CHR}, \mathrm{BKF}$, and I1P were the most important components, contributing most heavily to the joint effect, according to the PIPs (Table S9). Figure 1C showed the trends of exposure-response functions of the 10 PAHs. When all the other PAHs fixed at the median, PHE, BAA, CHR, and BKF showed a somewhat U-shaped pattern with NTD risk, I1P and BghiP showed a S-shaped trend with NTD risk, and FLO, BAP, and BBF showed no obvious trend with NTD risk. Possible interactive effects among different components in the PAH mixture were estimated, and no significant associations were obtained (Fig. 1D).

\subsection{Sensitivity analyses}

To test the robustness of our results, two sensitivity analyses were performed. Because gestational age was significantly higher in controls than in cases, and PAH concentrations decreased with the increase in gestational weeks (Table S10), gestational age could have been an important confounder in our analyses. Therefore, subgroup analyses were performed based on gestational age. Even in this smaller sample (gestational age $\geq 28$ weeks), the associations between levels of PAHs and the risk for spina bifida were similar to the overall results, and the direction of the associations between PAHs and NTDs was consistent with those in the total population (Table S11). Then, we performed sensitivity analyses with the median concentrations in the controls as the cut-off points, and the results were consistent with before in unadjusted logistic regression. Although the results turned to be not significant, the directions of the association were consistent with the main results (Table S12).

\section{Discussion}

In this study, we observed significant associations between levels of PAHs in umbilical cord tissue and the risk for NTDs. Using traditional logistic regression, we examined the single effects of exposure to individual PAHs on NTD risk. Using BKMR, we evaluated the joint effect of exposure to multiple PAHs on the risk for NTDs. Overall, exposure to both individual and multiple PAHs was associated with an increased risk for NTDs. 
Several population-based studies have examined the association between prenatal PAH exposure and risk for fetal NTDs based on questionnaires (Langlois et al., 2012; Li et al., 2011). One study conducted in Shanxi province of China evaluated indoor air pollution from coal combustion and found that women exposed to this pollution had a higher risk of having a pregnancy affected by NTDs (Li et al., 2011). Another study examined the association between periconceptional exposure to PAHs and risk for NTDs; the results suggested a positive association between maternal PAH exposure and fetal spina bifida in normal-weight or underweight women (Langlois et al., 2012). However, the estimates of exposure in these studies were based on participant self-report, which may have decreased their reliability or inaccuracy. Moreover, questionnaires can not include all sources of exposure, which makes it impossible to determine participants' true level of exposure. In this study, we used the levels of PAHs in umbilical cord tissue as biomarkers of in utero PAH exposure to accurately quantify PAH exposure from all sources.

Using logistic regression, we observed positive associations between levels of 10 PAHs in umbilical cord tissue and NTD risk in the unadjusted model, as well as positive associations between PHE, FLO, PYR, and BBF in the adjusted models. Using BKMR, which allows for testing both the joint effect of multiple PAHs and the single effects of individual PAHs within a mixture, we obtained a significant positive association between exposure to a combination of PAHs and risk for NTDs in this study population. This association was statistically significant when PAH concentrations in umbilical cord tissue were all above the 65th percentile compared to their median. Overall, our results are comparable to the results of previous studies based on PAH or PAH-DNA adducts in placental tissue, maternal serum, and cord blood in smaller populations in Shanxi province, China (Ren et al., 2011; Wang et al., 2015; Yi et al., 2015). The umbilical cord develops from the very beginning with the fetus and exists during the entire gestation; therefore, PAH concentrations in cord tissue may represent a relatively long period of exposure. In comparison, cord blood exchanges rapidly between the mother and the fetus, and PAH levels in cord blood may reflect short-term exposure. This study used PAH concentrations in umbilical cord tissue as markers of fetal exposure, which could better represent the exposure of the fetus compared to the markers in maternal blood or placental tissue, and therefore is an extension of previous studies.

PAHs are the most common and widely distributed persistent organic pollutants in the environment, and significant public concern has been raised over their harmful effects on human and animal health. The incomplete combustion of fossil fuels and biomass is the main anthropogenic source of PAH pollution. In addition, the incineration of municipal waste and agricultural nonsurface source emissions contribute to adverse health effects (Sun et al., 2021). These substances easily reach the fetus by crossing the placental barrier and may adversely influence the development of the fetus and the birth outcome (Ferguson et al., 2017; Huang et al., 2020). In humans, PAHs can be metabolized and transformed to produce reactive oxygen species, which can result in oxidative stress (Bortey-Sam et al., 2017; Cao et al., 2020; Ferguson et al., 2017; Zhang et al., 2020). Oxidative stress-induced DNA modification and lipid modification may adversely impact fetal neural tube development (Chang et al., 2003; James et al., 2004; Martín et al., 2004; Zhao et al., 2006). Additional in vitro or in vivo studies using animal models and other methods of assessing exposure and outcomes have indicated that excessive apoptosis in fetal central nervous tissue, oxidative stress-induced DNA damage, or aberrant DNA methylation may be the 
underlying mechanism in the development of NTDs (Huang et al., 2020; Lin et al., 2018; Lin et al., 2019; Wang et al., 2017), which supports our findings of associations between in uterus PAH exposure and risk for NTDs. These studies used single PAH or treated each of the multiple PAHs as an independent exposure. Our results revealed a positive association between multiple PAH exposure and risk for NTDs. Therefore, the synergistic effects of oxidative stress and/or aberrant epigenetic DNA modification were hypothesized to underlie their joint effects on increasing risk for NTDs (Sun et al., 2021).

To the best of our knowledge, no study has examined the change of PAH in umbilical cord tissue with gestation. Our results suggested that the concentrations of PAHs decreased with the increase in gestational week. One possible explanation is that the degradation of PAHs gets faster with the length of gestation since PAHs are mainly metabolized by cytochrome P450 (CYP450) enzymes in the liver. With the development of the liver with gestational age, the expression level of CYP450 is increased, and the metabolism of CYP450 to PAH is accelerated (von Koschembahr et al., 2020). Another possibility is that the umbilical cord grows faster compared to the accumulation of PAHs in the umbilical cord tissue. However, both speculations need to be tested in future studies.

This study had several strengths. First, as far as we know, this is the first study to examine the association between PAH exposure and NTD risk using PAH concentrations in umbilical cord tissue as biomarkers of internal exposure. Compared to markers in maternal blood or placental tissue, markers in the umbilical cord should better reflect fetal exposure. Second, we used BKMR, which is a novel, flexible statistical method, to examine the association between PAH exposure and risk for NTDs, which allowed us to quantify the joint effect of a PAH mixture and visualize the trend. Moreover, by using a statistical model suited to assessing combined exposure, we add contextual understanding to the effect of exposure to multiple pollutants on risk for fetal NTDs. These strengths notwithstanding, there were also some limitations to the study. First, the sample size was small, which limited our ability to perform more detailed analyses by the subtypes of NTDs. Second, because of the nature of the outcome and the casecontrol design, PAH concentrations in umbilical cord tissue may not reflect true PAH exposure during neural tube development exactly. This problem can only be resolved with large-scale cohort studies.

\section{Conclusions}

Using PAH content in umbilical cord tissue as biomarkers of fetal exposure, this study revealed that in utero exposure to higher levels of multiple PAHs is associated with an increased risk for NTDs. Avoiding exposure to PAHs before or during early pregnancy may help reduce the risk for NTDs in the fetus.

\section{Declarations}

\section{Author contributions}

Lei Jin and Aiguo Ren conceived the study; Jinhui Yu, Zaiming Su, Mingkun Tong, Yali Zhang, Jufen Liu, Linlin Wang and Zhiwen Li collect data and tissue sample; Yin Sun performed the experiments; Shengju 
Yin and Yin Sun analyzed, interpreted the data, and wrote the paper; Aiguo Ren supervised the study

Conflict of interest The authors declare that they have no known competing fnancial interests or personal relationships that could have appeared to infuence the work reported in this paper.

Ethical Approval The study was approved by the institutional review board of Peking University.

Consent to Participate Each mother signed a written consent to participate in the study.

Data Availability All data are available in our paper or from the corresponding author on reasonable request.

\section{Acknowledgments}

This work was supported by the National Key Research and Development Program, Ministry of Science and Technology of the People's Republic of China (Grant Nos 2018YFC1004300, 2018YFC1004301, and 2016YFC1000501). We express our gratitude to other investigators and the local healthcare workers in Shanxi Province, China, for their help with data and biospecimen collection during the study. We also thank the women who participated in the study.

Appendix A: Supplementary Information

\section{References}

Barbieri, O., et al., 1986. Embryotoxicity of benzo(a)pyrene and some of its synthetic derivatives in Swiss mice. Cancer Res. 46, 94-98.

Blencowe, H., et al., 2018. Estimates of global and regional prevalence of neural tube defects for 2015: a systematic analysis. Ann N Y Acad Sci. 1414, 31-46.

Bobb, J. F., et al., 2018. Statistical software for analyzing the health effects of multiple concurrent exposures via Bayesian kernel machine regression. Environ. Health. 17, 67.

Bobb, J. F., et al., 2015. Bayesian kernel machine regression for estimating the health effects of multipollutant mixtures. Biostatistics (Oxford, England). 16, 493-508.

Bókony, V., et al., 2018. Endocrine disruptors in breeding ponds and reproductive health of toads in agricultural, urban and natural landscapes. Sci Total Environ. 634, 1335-1345.

Bortey-Sam, N., et al., 2017. Oxidative stress and respiratory symptoms due to human exposure to polycyclic aromatic hydrocarbons (PAHs) in Kumasi, Ghana. Environ Pollut. 228, 311-320.

Cao, L., et al., 2020. Oxidative damage mediates the association between polycyclic aromatic hydrocarbon exposure and lung function. Environ Health. 19, 75. 
Chang, T. I., et al., 2003. Oxidant regulation of gene expression and neural tube development: Insights gained from diabetic pregnancy on molecular causes of neural tube defects. Diabetologia. 46, 538-545.

Copp, A. J., et al., 2013. Neural tube defects: recent advances, unsolved questions, and controversies. Lancet Neurol. 12, 799-810.

Drwal, E., et al., 2019. Review: Polycyclic aromatic hydrocarbons (PAHs)-Action on placental function and health risks in future life of newborns. Toxicology. 411, 133-142.

Ferguson, K. K., et al., 2017. Urinary Polycyclic Aromatic Hydrocarbon Metabolite Associations with Biomarkers of Inflammation, Angiogenesis, and Oxidative Stress in Pregnant Women. Environ Sci Technol. 51, 4652-4660.

Greene, N. D., Copp, A. J., 2014. Neural tube defects. Annu Rev Neurosci. 37, 221-42.

Huang, Y., et al., 2019a. Decreased global DNA hydroxymethylation in neural tube defects: Association with polycyclic aromatic hydrocarbons. Epigenetics. 14, 1019-1029.

Huang, Y., et al., 2020. Neural Tube Defects and ZIC4 Hypomethylation in Relation to Polycyclic Aromatic Hydrocarbon Exposure. Front Cell Dev Biol. 8, 582661.

Huang, Y., et al., 2019b. Casp8 hypomethylation and neural tube defects in association with polycyclic aromatic hydrocarbon exposure. Clin Epigenetics. 11, 72.

James, S. J., et al., 2004. Metabolic biomarkers of increased oxidative stress and impaired methylation capacity in children with autism. Am J Clin Nutr. 80, 1611-1617.

Kamelia, L., et al., 2019. Prenatal developmental toxicity testing of petroleum substances using the zebrafish embryotoxicity test. Altex. 36, 245-260.

Kim, K. H., et al., 2013. A review of airborne polycyclic aromatic hydrocarbons (PAHs) and their human health effects. Environ Int. 60, 71-80.

Kondo, A., et al., 2017. Neural tube defects: Risk factors and preventive measures. Congenit Anom (Kyoto). 57, 150-156.

Langlois, P. H., et al., 2012. Maternal occupational exposure to polycyclic aromatic hydrocarbons and risk of neural tube defect-affected pregnancies. Birth Defects Res A Clin Mol Teratol. 94, 693-700.

$\mathrm{Li}$, Z., et al., 2011. Indoor air pollution from coal combustion and the risk of neural tube defects in a rural population in Shanxi Province, China. Am J Epidemiol. 174, 451-458.

Lin, S., et al., 2018. Oxidative Stress and Apoptosis in Benzo[a]pyrene-Induced Neural Tube Defects. Free Radic Biol Med. 116, 149-158. 
Lin, S., et al., 2019. Aberrant methylation of Pax3 gene and neural tube defects in association with exposure to polycyclic aromatic hydrocarbons. Clin Epigenetics. 11, 13.

Mark-Kappeler, C. J., et al., 2011. Xenobiotic effects on ovarian preantral follicles. Biol Reprod. 85, 871883.

Martín, I., et al., 2004. Oxidative stress in mothers who have conceived fetus with neural tube defects: the role of aminothiols and selenium. Clin Nutr. 23, 507-514.

$\mathrm{Ni}, \mathrm{W}$. , et al., 2019. Levels of polycyclic aromatic hydrocarbons in umbilical cord and risk of orofacial clefts. Sci Total Environ. 678, 123-132.

Nikolopoulou, E., et al., 2017. Neural tube closure: cellular, molecular and biomechanical mechanisms. Development. 144, 552-566.

Nisbet, I. C., LaGoy, P. K., 1992. Toxic equivalency factors (TEFs) for polycyclic aromatic hydrocarbons (PAHs). Regul Toxicol Pharmacol. 16, 290-300.

Oliveira, M., et al., 2019. Children environmental exposure to particulate matter and polycyclic aromatic hydrocarbons and biomonitoring in school environments: A review on indoor and outdoor exposure levels, major sources and health impacts. Environ Int. 124, 180-204.

Ren, A., et al., 2011. Association of selected persistent organic pollutants in the placenta with the risk of neural tube defects. Proc. Natl. Acad. Sci. USA. 108, 12770-12775.

Stogiannidis, E., Laane, R., 2015. Source characterization of polycyclic aromatic hydrocarbons by using their molecular indices: an overview of possibilities. Rev Environ Contam Toxicol. 234, 49-133.

Sun, K., et al., 2021. A review of human and animals exposure to polycyclic aromatic hydrocarbons: Health risk and adverse effects, photo-induced toxicity and regulating effect of microplastics. Sci Total Environ. 773, 145403.

von Koschembahr, A., et al., 2020. Metabolism and genotoxicity of polycyclic aromatic hydrocarbons in human skin explants: mixture effects and modulation by sunlight. Arch Toxicol. 94, 495-507.

Wang, B., et al., 2015. Levels of polycyclic aromatic hydrocarbons in maternal serum and risk of neural tube defects in offspring. Environ Sci Technol. 49, 588-596.

Wang, L., et al., 2017. Apoptosis, Expression of PAX3 and P53, and Caspase Signal in Fetuses with Neural Tube Defects. Birth Defects Res. 109, 1596-1604.

Waring, R. H., et al., 2016. In utero exposure to carcinogens: Epigenetics, developmental disruption and consequences in later life. Maturitas. 86, 59-63. 
Wilde, J. J., et al., 2014. Genetic, epigenetic, and environmental contributions to neural tube closure. Annu Rev Genet. 48, 583-611.

Yi, D., et al., 2015. Levels of PAH-DNA adducts in cord blood and cord tissue and the risk of fetal neural tube defects in a Chinese population. Neurotoxicology. 46, 73-78.

Yin, S., et al., 2021. Prenatal exposure to organochlorine pesticides is associated with increased risk for neural tube defects. Sci Total Environ. 770, 145284.

Zhang, H., et al., 2020. Association of internal exposure to polycyclic aromatic hydrocarbons with inflammation and oxidative stress in prediabetic and healthy individuals. Chemosphere. 253, 126748.

Zhao, W., et al., 2006. Neural tube defects and maternal biomarkers of folate, homocysteine, and glutathione metabolism. Birth Defects Res A Clin Mol Teratol. 76, 230-236.

\section{Figures}
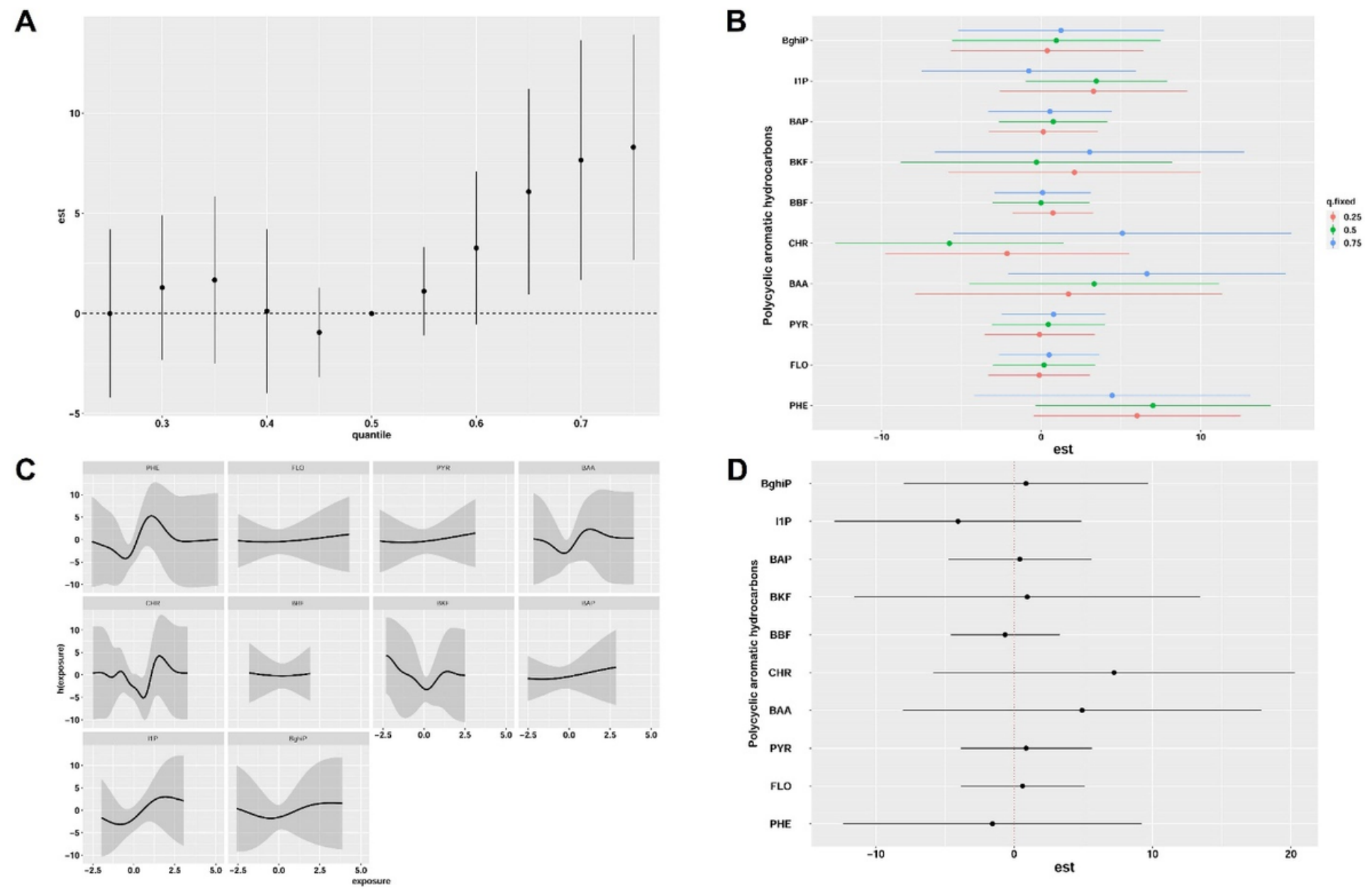

Figure 1 
Joint effect of PAH mixture on NTD risk estimated by Bayesian kernel machine regression. (A) Overall effect of the 10 PAHs on the risk for NTDs. This panel plots the difference in risk for NTDs when concentrations of all PAHs are fixed at a specific percentile compared to their median. (B) Single effects of individual PAHs on the risk for NTDs. This panel plots the change in risk for NTDs when an individual $\mathrm{PAH}$ is at the 75 th vs. 25 th percentile when all other PAHs are fixed at a specific quantile (25th, 50th, or 75th). (C) Univariate exposure-response functions and 95\% credible intervals for each PAH when the other PAHs are fixed at their median. (D) Interactive effects, defined as the change in single-PAH health effects when all other PAHs are fixed at their 25th percentile compared to their 75th percentile.

Abbreviations: PAH, polycyclic aromatic hydrocarbon; NTD, neural tube defect; PHE, phenanthrene; FLO, fluoranthene; PYR, pyrene; BAA, benzo(a)anthracene; CHR, chrysene; BBF, benzo(b)fluoranthene; BKF, benzo(k)fluoranthene; BAP, benzo(a)pyrene; I1P, Indeno(1,2,3-cd)pyrene; BghiP, benzo(g,h,i)perylene.

\section{Supplementary Files}

This is a list of supplementary files associated with this preprint. Click to download.

- Supplementarymaterial.docx 